\title{
ON THE COMMUTATORS OF SINGULAR INTEGRALS RELATED TO BLOCK SPACES
}

\author{
SHANZHEN LU*AND HUOXIONG WU
}

\begin{abstract}
In this paper, the commutators of singular integrals with rough kernels are considered. By the method of block decomposition for kernel function and Fourier transform estimates, some new results about the $L^{p}\left(\mathbb{R}^{n}\right)$ boundedness for these commutators are obtained.
\end{abstract}

\section{$\S 1$. Introduction}

Let $\mathbb{R}^{n}, n \geq 2$, be the $n$-dimensional Euclidean space and $S^{n-1}$ be the unit sphere in $\mathbb{R}^{n}$ equipped with the normalized Lebesgue measure $d \sigma=d \sigma\left(x^{\prime}\right)$. Let $\Omega(x)$ be a homogeneous function of degree zero and have mean value zero on $S^{n-1}$. Suppose that $h(t) \in L^{\infty}(0, \infty)$. Define the singular integral operator $T$ by

$$
T f(x)=p . v \cdot \int_{\mathbb{R}^{n}} \frac{\Omega(x-y)}{|x-y|^{n}} h(|x-y|) f(y) d y .
$$

For a positive integer $k$ and $a(x) \in B M O\left(\mathbb{R}^{n}\right)$, define the $k$-th order commutator $T_{a, k}$ generated by $T$ and $a$

$$
T_{a, k} f(x)=T\left((a(x)-a(\cdot))^{k} f\right)(x), f \in C_{0}^{\infty}\left(\mathbb{R}^{n}\right) .
$$

It was proved by Coifman, Rochberg and Weiss [4] that if $\Omega \in$ $\operatorname{Lip}_{\alpha}\left(S^{n-1}\right)(0<\alpha \leq 1)$ and $h \equiv 1$, then $T_{a, 1}$ is bounded on $L^{p}\left(\mathbb{R}^{n}\right)$ with bound $C\|a\|_{B M O\left(\mathbb{R}^{n}\right)}$ for $1<p<\infty$. Afterwards, by a well-known result of Duoandikoetxea [6] and the boundedness criterion of Alvarez-Bagby-KurtzPérez for the commutators of linear operator (see [2]), we have obtained the following theorem (see also [10]):

Received June 12, 2002.

Revised January 7, 2003, February 25, 2003.

2000 Mathematics Subject Classification: 42B20, 47B20, 42B35, 47A30.

${ }^{*}$ The first author is supported by the National 973 project and the SEDF of China. 
TheOREm A. ([6, 2, 10]) Let $\Omega, a, k$ be as above and $h \equiv 1,1<p<$ $\infty$. If $\Omega \in \cup_{q>1} L^{q}\left(S^{n-1}\right)$, then $T_{a, k}$ is bounded on $L^{p}\left(\mathbb{R}^{n}\right)$.

Recently, to weaken the condition imposed on $\Omega, \mathrm{Hu}$ Guoen et al. employed the method of Littlewood-Paley theory and Fourier transform estimates from [7] to obtain the following results.

Theorem B. Let $\Omega, a, k$ be as above. Then $T_{a, k}$ is bounded on $L^{p}\left(\mathbb{R}^{n}\right)$ with bound $C\|a\|_{B M O\left(\mathbb{R}^{n}\right)}^{k}$, if one of the following conditions holds.

(i) $($ see $[12]) . p=2, h \equiv 1, \Omega \in L\left(\log ^{+} L\right)^{k+1}\left(S^{n-1}\right)$.

(ii) (see [9]). $\quad p=2, h \equiv 1$ and for some $\alpha>k+1$, $\Omega$ satisfies

$$
\sup _{\xi \in S^{n-1}} \int_{S^{n-1}}|\Omega(\theta)|\left(\log \frac{1}{|\theta \cdot \xi|}\right)^{\alpha} d \theta .
$$

(iii) (see [13] or [9]). For some $\alpha>k+1, \Omega \in L\left(\log ^{+} L\right)^{\alpha}\left(S^{n-1}\right)$ and for some $s>1, h$ satisfies $\sup _{R>0} \int_{R}^{2 R}|h(r)|^{s} r^{-1} d r<\infty, 2 \alpha /(2 \alpha-(k+1))<$ $p<2 \alpha /(k+1)$ or $p=2$.

Theorem B certainly improve Theorem A since both the condition $\Omega \in L\left(\log ^{+} L\right)^{\alpha}\left(S^{n-1}\right)(\alpha>k+1)$ and the size condition (1.3) are properly weaker than the condition $\Omega \in \bigcup_{q>1} L^{q}\left(S^{n-1}\right)$. Unfortunately, the condition on $\Omega$ in Theorem B greatly depends on the order $k$ of $T_{a, k}$. It is natural to ask whether there exists a weaker size condition on $\Omega$, which is independent of $k$, such that $T_{a, k}$ is bounded on $L^{p}\left(\mathbb{R}^{n}\right), 1<p<\infty$. The main purpose of this paper is to give a positive answer to this problem. Inspired by [1], we shall show that $T_{a, k}$ is bounded on $L^{p}\left(\mathbb{R}^{n}\right)$ for $1<p<\infty$, if $\Omega \in B_{q}^{0,0}\left(S^{n-1}\right)$ for some $q>1$. Here $B_{q}^{0,0}\left(S^{n-1}\right)$ denotes certain block spaces introduced by Jiang and Lu(see [15]). We remark that some ideas in the proof of our main results are taken from [7] and [11]. Before stating the main results, we briefly review some pertinent concepts.

Definition 1. ([15]) A $q$-block on $S^{n-1}$ is an $L^{q}(1<q \leq \infty)$ function $b(\cdot)$ that satisfies

$$
\text { (i) } \operatorname{supp}(b) \subseteq Q, \quad \text { (ii) }\|b\|_{L^{q}\left(S^{n-1}\right)} \leq|Q|^{\frac{1}{q}-1}
$$

where $Q=S^{n-1} \cap\left\{y \in \mathbb{R}^{n}:|y-\varsigma|<\rho\right.$ for some $\varsigma \in S^{n-1}$ and $\rho \in$ $(0,1]\}$. 
Definition 2. ([15]) The block spaces $B_{q}^{0,0}$ on $S^{n-1}$ are defined by

$$
B_{q}^{0,0}\left(S^{n-1}\right)=\left\{\Omega \in L^{1}\left(S^{n-1}\right): \Omega\left(y^{\prime}\right)=\sum_{s} C_{s} b_{s}\left(y^{\prime}\right), M_{q}^{0,0}\left(\left\{C_{s}\right\}\right)<\infty\right\}
$$

where each $C_{s}$ is a complex number, each $b_{s}$ is a $q$-block supported in $Q_{s}$, and

$$
M_{q}^{0,0}\left(\left\{C_{s}\right\}\right)=\sum_{s}\left|C_{s}\right|\left\{1+\log ^{+} \frac{1}{\left|Q_{s}\right|}\right\}
$$

It should be pointed out that the method of block decomposition for functions was invented by Taibleson and Weiss [17] in the study of the convergence of the Fourier series. Later on, many application of the block decomposition to harmonic analysis were discovered (see [1], [14]-[16] etc.). For further background and information about the theory of spaces generated by blocks and its applications to harmonic analysis, one can consult the book [15]. In [14], Keitoku and Sato showed that for any $q>1$,

$$
\bigcup_{r>1} L^{r}\left(S^{n-1}\right) \subset B_{q}^{0,0}\left(S^{n-1}\right)
$$

which is a proper inclusion. And from [14], we easily see that $B_{q}^{0,0}\left(S^{n-1}\right)$ is not contained in $L\left(\log ^{+} L\right)^{1+\varepsilon}\left(S^{n-1}\right)$ for any $\varepsilon>0$ although the relationship between $B_{q}^{0,0}\left(S^{n-1}\right)$ and $L \log ^{+} L\left(S^{n-1}\right)$ remains open.

Definition 3. ([3]) A locally integrable function $a(x)$ will be said to belong to $B L O\left(\mathbb{R}^{n}\right)$, if there is a constant $C$ such that for any cube $Q$

$$
m_{Q}(a)-\inf _{x \in Q} a(x) \leq C,
$$

where $m_{Q}(a)=|Q|^{-1} \int_{Q} a(x) d x$.

If $a \in B L O\left(\mathbb{R}^{n}\right)$, then we denote $\|a\|_{B L O\left(\mathbb{R}^{n}\right)}=\sup _{Q}\left\{m_{Q}(a)-\right.$ $\left.\inf _{x \in Q} a(x)\right\}$.

Obviously, $L^{\infty}\left(\mathbb{R}^{n}\right) \subset B L O\left(\mathbb{R}^{n}\right) \subset B M O\left(\mathbb{R}^{n}\right)$ and if $a \in B L O\left(\mathbb{R}^{n}\right)$, then

$$
\|a\|_{B M O\left(\mathbb{R}^{n}\right)} \leq 2\|a\|_{B L O\left(\mathbb{R}^{n}\right)}
$$

Now let us formulate our main results. 
THEOREM 1. Let $\Omega$ be homogeneous of degree zero and have mean value zero, $k$ be a positive integer and $a \in B M O\left(\mathbb{R}^{n}\right)$. If $h(t) \in L^{\infty}(0, \infty)$ and $\Omega \in B_{q}^{0,0}\left(S^{n-1}\right)$ for $q>1$, then the commutator $T_{a, k}$ is bounded on $L^{2}\left(\mathbb{R}^{n}\right)$ with bound $C\|a\|_{B M O\left(\mathbb{R}^{n}\right)}^{k}$.

For the case of $p \neq 2,1<p<\infty$, we need to impose some restrictions on BMO functions $a(x)$ as follows.

Theorem 2. Let $\Omega, h, k$ be as in Theorem $1,1<p<\infty$. If $a \in$ $B L O\left(\mathbb{R}^{n}\right)$ and $a(x)$ is subharmonic, then $T_{a, k}$ is bounded on $L^{p}\left(\mathbb{R}^{n}\right)$ with bound $C\|a\|_{B L O\left(\mathbb{R}^{n}\right)}^{k}$.

Remark 1. It is worth pointing out that a BMO function $a(x)$ satisfying the restrictive conditions in Theorem 2 exists. A typical example is $\log |x|$.

Remark 2. $\bigcup_{r>1} L^{r}\left(S^{n-1}\right)$ is properly contained in $B_{q}^{0,0}\left(S^{n-1}\right)$ for any $q>1$, and $B_{q}^{0,0}\left(S^{n-1}\right)$ is independent of the order of $T_{a, k}$ and is not contained in $L\left(\log ^{+} L\right)^{\alpha}\left(S^{n-1}\right)(\alpha>1)$. Therefore our theorems are an essential improvement on Theorem A and an great extension of the result in Theorem B.

In proving Theorem 2, we shall use the following $L^{p}$-boundedness of $M_{a, k}^{\Omega}$, a maximal operator related to higher order commutators, defined by

$$
M_{a, k}^{\Omega} f(x)=\sup _{r>0} \frac{1}{r^{n}} \int_{|x-y|<r}|a(x)-a(y)|^{k}|h(|x-y|) \Omega(x-y) f(y)| d y .
$$

THEOREM 3. Under the same hypothesis as in Theorem 2, the operator $M_{a, k}^{\Omega}$ satisfies

$$
\left\|M_{a, k}^{\Omega} f\right\|_{p} \leq C\|a\|_{B L O\left(\mathbb{R}^{n}\right)}^{k}\|f\|_{p}
$$

Throughout this paper, $C$ always denotes positive constants that are independent of the essential variables but whose value may vary at each occurrence. 


\section{§2. Proof of Theorem 1}

Let us begin with some preliminary lemmas.

LEMma 1. ([11]) Let $\phi \in C_{0}^{\infty}\left(\mathbb{R}^{n}\right)$ be a radial function such that $\operatorname{supp} \phi \subset\{1 / 4 \leq|\xi| \leq 4\}$ and

$$
\sum_{l \in \mathbb{Z}} \phi^{3}\left(2^{-l} \xi\right)=1,|\xi| \neq 0 .
$$

Denote by $S_{l}$ the multiplier operator

$$
\widehat{S_{l} f}(\xi)=\phi\left(2^{-l} \xi\right) \hat{f}(\xi)
$$

and $S_{l}^{2} f(x)=S_{l}\left(S_{l} f\right)(x)$. For any positive integer $k$ and $a \in B M O\left(\mathbb{R}^{n}\right)$, consider the $k$-th order commutator of $S_{l}$ and $S_{l}^{2}$, respectively, defined by

$$
S_{l ; a, k} f(x)=S_{l}\left((a(x)-a(\cdot))^{k} f\right)(x), \quad f \in C_{0}^{\infty}\left(\mathbb{R}^{n}\right)
$$

and

$$
S_{l ; a, k}^{2} f(x)=S_{l}^{2}\left((a(x)-a(\cdot))^{k} f\right)(x), \quad f \in C_{0}^{\infty}\left(\mathbb{R}^{n}\right) .
$$

Then for all $1<p<\infty$,

$$
\begin{aligned}
& \text { (a) }\left\|\left(\sum_{l \in \mathbb{Z}}\left|S_{l ; a, k} f\right|^{2}\right)^{\frac{1}{2}}\right\|_{p} \leq C\|a\|_{B M O\left(\mathbb{R}^{n}\right)}^{k}\|f\|_{p} ; \\
& \text { (b) }\left\|\left(\sum_{l \in \mathbb{Z}}\left|S_{l ; a, k}^{2} f\right|^{2}\right)^{\frac{1}{2}}\right\|_{p} \leq C\|a\|_{B M O\left(\mathbb{R}^{n}\right)}^{k}\|f\|_{p} ; \\
& \text { (c) }\left\|\sum_{l \in \mathbb{Z}} S_{l ; a, k} f_{l}\right\|_{p} \leq C(n, k, p)\|a\|_{B M O\left(\mathbb{R}^{n}\right)}^{k}\left\|\left(\sum_{l \in \mathbb{Z}}\left|f_{l}\right|^{2}\right)^{\frac{1}{2}}\right\|_{p}, \\
& \quad f_{l} \in C_{0}^{\infty}\left(\mathbb{R}^{n}\right)(l \in \mathbb{Z}) .
\end{aligned}
$$

LEMma 2. ([11]) Let $0<\delta<\infty$, and take a function $m_{\delta} \in C_{0}^{\infty}\left(\mathbb{R}^{n}\right)$ with support contained in $\left\{\xi \in \mathbb{R}^{n}:|\xi| \leq \delta\right\}$. Suppose that for some positive constant $\alpha$,

$$
\left\|m_{\delta}\right\|_{\infty} \leq C \min \left\{\delta^{\alpha}, \delta^{-\alpha}\right\}, \quad\left\|\nabla m_{\delta}\right\|_{\infty} \leq C .
$$

Let $T_{\delta}$ be the multiplier operator defined by

$$
\widehat{T_{\delta} f}(\xi)=m_{\delta}(\xi) \hat{f}(\xi)
$$


For a positive integer $k$ and $a \in B M O\left(\mathbb{R}^{n}\right)$, let $T_{\delta ; a, k}$ be the $k$-th order commutator of $T_{\delta}$. Then for any fixed $0<v<1$, there exists a positive constant $C=C(n, k, v)$ such that

$$
\left\|T_{\delta ; a, k} f\right\|_{2} \leq C \min \left\{\delta^{\alpha v}, \delta^{-\alpha v}\right\}\|a\|_{B M O\left(\mathbb{R}^{n}\right)}^{k}\|f\|_{2} .
$$

Lemma 3. Let $\Omega\left(x^{\prime}\right)=\sum_{s} C_{s} b_{s}\left(x^{\prime}\right), h(t)$ be as in Theorem 1. For $j \in \mathbb{Z}$, set

$$
\begin{aligned}
K_{j}(x) & =\frac{\Omega(x)}{|x|^{n}} h(|x|) \chi_{\left\{2^{j} \leq|x|<2^{j+1}\right\}}(x), \\
K_{j, s}(x) & =\frac{b_{s}(x)}{|x|^{n}} h(|x|) \chi_{\left\{2^{j} \leq|x|<2^{j+1}\right\}}(x),
\end{aligned}
$$

and $m_{j}(\xi)=\widehat{K_{j}}(\xi), m_{j, s}(\xi)=\widehat{K_{j, s}}(\xi)$. Then we have

(i) $\left|m_{j}(\xi)\right| \leq C\left|2^{j} \xi\right|$;

(ii) $\left|m_{j, s}(\xi)\right| \leq\left|2^{j} \xi\right|^{\frac{1}{2 \log \left|Q_{s}\right|}}$, if $\left|Q_{s}\right|<e^{\frac{q}{1-q}}$;

(iii) $\left|m_{j, s}(\xi)\right| \leq C\left|2^{j} \xi\right|^{-\omega}$, if $\left|Q_{s}\right| \geq e^{\frac{q}{1-q}}$.

Here $C$ and $\omega$ are positive constants independent of $j, s, \xi$ and $b_{s}$.

Proof. By the mean zero property and the integrability of $\Omega$ on $S^{n-1}$, we have

$$
\begin{aligned}
\left|m_{j}(\xi)\right| & =\left.\left|\int_{2^{j} \leq|y|<2^{j+1}} h(|y|)\right| y\right|^{-n} \Omega\left(y^{\prime}\right) e^{-2 \pi i y \cdot \xi} d y \mid \\
& =\left|\int_{2^{j}}^{2^{j+1}} h(t) t^{-1} \int_{S^{n-1}} \Omega\left(y^{\prime}\right) e^{-2 \pi i t y^{\prime} \cdot \xi} d \sigma\left(y^{\prime}\right) d t\right| \\
& \leq C \int_{2^{j}}^{2^{j+1}} t^{-1}\left|\int_{S^{n-1}} \Omega\left(y^{\prime}\right)\left(e^{-2 \pi i t y^{\prime} \cdot \xi}-1\right) d \sigma\left(y^{\prime}\right)\right| d t \\
& \leq C \int_{2^{j}} t^{-1} \int_{S^{n-1}}\left|\Omega\left(y^{\prime}\right)\right|\left|2 \pi t y^{\prime} \cdot \xi\right| d \sigma\left(y^{\prime}\right) d t \\
& \leq C\|\Omega\|_{L^{1}\left(S^{n-1}\right)}|\xi| \int_{2^{j}}^{2^{j+1}} d t \leq C\left|2^{j} \xi\right| .
\end{aligned}
$$

Thus, (i) is proved. (ii) and (iii) are the special cases of (ii) and (iii) Lemma 2.2 in [1]. The proof of Lemma 3 is complete. 
Proof of Theorem 1. For $j \in \mathbb{Z}$, let $K_{j}(\xi), m_{j}(\xi)$ be as in Lemma 3 and $\phi$ be as in Lemma 1. Define the multiplier operator $S_{l}$ by

$$
\widehat{S_{l} f}(\xi)=\phi\left(2^{-l} \xi\right) \hat{f}(\xi) .
$$

Set $m_{j}^{l}(\xi)=m_{j}(\xi) \phi\left(2^{j-l} \xi\right)$ and $\widehat{T_{j}^{l} f}(\xi)=m_{j}^{l}(\xi) \hat{f}(\xi)$. Let

$$
U_{l} f(x)=\sum_{j \in \mathbb{Z}}\left(\left(S_{l-j} T_{j}^{l} S_{l-j}\right)_{a, k} f\right)(x) .
$$

We know from [11] that for $f, g \in C_{0}^{\infty}\left(\mathbb{R}^{n}\right)$,

$$
\int_{\mathbb{R}^{n}} g(x) T_{a, k} f(x) d x=\int_{\mathbb{R}^{n}} g(x) \sum_{l \in \mathbb{Z}} U_{l} f(x) d x .
$$

Hence

$$
\left\|T_{a, k} f\right\|_{2} \leq \sum_{l \in \mathbb{Z}}\left\|U_{l} f\right\|_{2}
$$

With the aid of the formula

$$
(a(x)-a(y))^{k}=\sum_{m=0}^{k} C_{k}^{m}(a(x)-a(z))^{m}(a(z)-a(y))^{k-m}, x, y, z \in \mathbb{R}^{n},
$$

we get

$$
\begin{aligned}
\int_{\mathbb{R}^{n}} g(x) U_{l} f(x) d x \\
\quad=\sum_{m=0}^{k} C_{k}^{m} \int_{\mathbb{R}^{n}} g(x) \sum_{j \in \mathbb{Z}} S_{l-j ; a, k-m}\left(\left(T_{j}^{l} S_{j-l}\right)_{a, m} f\right)(x) d x,
\end{aligned}
$$

for $f, g \in C_{0}^{\infty}\left(\mathbb{R}^{n}\right)$ by a straightforward computation.

By Lemma 1(c), we get

$$
\begin{aligned}
\left\|U_{l} f\right\|_{2} & \leq C \sum_{m=0}^{k}\left\|\sum_{j \in \mathbb{Z}} S_{j-l ; a, k-m}\left(\left(T_{j}^{l} S_{l-j}\right)_{a, m} f\right)\right\|_{2} \\
& \leq C \sum_{m=0}^{k}\|a\|_{B O M\left(\mathbb{R}^{n}\right)}^{k-m}\left\|\left(\sum_{j \in \mathbb{Z}}\left|\left(T_{j}^{l} S_{l-j}\right)_{a, m} f\right|^{2}\right)^{\frac{1}{2}}\right\|_{2} .
\end{aligned}
$$


Case 1. We first consider the $L^{2}$-boundedness of $U_{l}$ for $l \leq 0$.

Let $\tilde{T}_{j}^{l}$ be the operator defined by

$$
\widehat{\tilde{T}_{j}^{l} f}(\xi)=m_{j}^{l}\left(2^{-j} \xi\right) \hat{f}(\xi) .
$$

By the vanishing moment and the integrability of $\Omega$, we have

$$
\left|\widehat{K}_{j}(\xi)\right| \leq C\left|2^{j} \xi\right|, \quad\left\|\nabla \widehat{K}_{j}\right\|_{\infty} \leq C 2^{j}
$$

Thus

$$
\left\|m_{j}^{l}\left(2^{-j} \cdot\right)\right\|_{\infty} \leq C 2^{l}, \quad\left\|\nabla m_{j}^{l}\left(2^{-j} \cdot\right)\right\|_{\infty} \leq C .
$$

Using this and Lemma 2, we obtain that for any fixed $0<v<1$ and positive integer $i$,

$$
\left\|\tilde{T}_{j ; a, i}^{l} f\right\|_{2} \leq C 2^{v l}\|a\|_{B M O\left(\mathbb{R}^{n}\right)}^{i}\|f\|_{2},
$$

which by dilation-invariance implies

$$
\left\|T_{j ; a, i}^{l} f\right\|_{2} \leq C 2^{v l}\|a\|_{B M O\left(\mathbb{R}^{n}\right)}^{i}\|f\|_{2} .
$$

On the other hand, the Plancherel theorem tells us that

$$
\left\|T_{j}^{l} f\right\|_{2} \leq C 2^{l}\|f\|_{2}
$$

Observe that for $f, g \in C_{0}^{\infty}\left(\mathbb{R}^{n}\right)$,

$$
\int_{\mathbb{R}^{n}} g(x)\left(T_{j}^{l} S_{l-j}\right)_{a, m} f(x) d x=\sum_{i=0}^{m} C_{m}^{i} \int_{\mathbb{R}^{n}} g(x) T_{j ;, a, i}^{l}\left(S_{l-j ; a, m-i} f\right)(x) d x .
$$

It follows from $(2.3),(2.4)$ and Lemma 1(a) that

$$
\begin{aligned}
\left\|\left(\sum_{j \in \mathbb{Z}}\left|\left(T_{j}^{l} S_{l-j}\right)_{a, m} f\right|^{2}\right)^{\frac{1}{2}}\right\|_{2}^{2} \\
\leq C \sum_{i=0}^{m}\left\|\left(\sum_{j \in \mathbb{Z}}\left|T_{j ; a, i}^{l}\left(S_{l-j ; a, m-i} f\right)\right|^{2}\right)^{\frac{1}{2}}\right\|_{2}^{2} \\
\leq C 2^{2 v l} \sum_{i=0}\|a\|_{B M O\left(\mathbb{R}^{n}\right)}^{2 i} \sum_{j \in \mathbb{Z}}\left\|S_{l-j ; a, m-i} f\right\|_{2}^{2} \\
\leq C 2^{2 v l}\|a\|_{B M O\left(\mathbb{R}^{n}\right)}^{2 m}\|f\|_{2}^{2}, \quad f \in C_{0}^{\infty}\left(\mathbb{R}^{n}\right) .
\end{aligned}
$$


Therefore

$$
\left\|U_{l} f\right\|_{2} \leq C 2^{v l}\|a\|_{B M O\left(\mathbb{R}^{n}\right)}^{k}\|f\|_{2}
$$

Case 2. Next we consider the $L^{2}$-estimate of $U_{l}$ for $l>0$.

Let $K_{j, s}, m_{j, s}$ be as in Lemma 3. Then $K_{j}(\xi)=\sum_{s} C_{s} K_{j, s}(\xi)$. Define the operator $T_{j}^{l, s}$ by

$$
\widehat{T_{j}^{l, s} f}(\xi)=\widehat{K_{j, s}}(\xi) \phi\left(2^{j-l} \xi\right) \hat{f}(\xi) .
$$

Then

$$
\begin{aligned}
T_{j}^{l} f(\xi) & =\sum_{s} C_{s} T_{j}^{l, s} f(\xi), \\
\left(T_{j}^{l} S_{l-j}\right)_{a, m} f(x) & =\sum_{s} C_{s}\left(T_{j}^{l, s} S_{l-j}\right)_{a, m} f(x) .
\end{aligned}
$$

And

$$
U_{l} f(x)=\sum_{s} C_{s} U_{l}^{s} f(x)
$$

where

$$
U_{l}^{s} f(x)=\sum_{j \in \mathbb{Z}}\left(S_{l-j} T_{j}^{l, s} S_{l-j}\right)_{a, k} f(x) .
$$

So

$$
\left\|U_{l} f\right\|_{2} \leq \sum_{s}\left|C_{s}\right|\left\|U_{l}^{s} f\right\|_{2}
$$

Similarly to (2.2), we have

$$
\left\|U_{l}^{s} f\right\|_{2} \leq C \sum_{m=0}^{k}\|a\|_{B M O\left(\mathbb{R}^{n}\right)}^{k-m}\left\|\left(\sum_{j \in \mathbb{Z}}\left|\left(T_{j}^{l, s} S_{l-j}\right)_{a, m} f\right|^{2}\right)^{\frac{1}{2}}\right\|_{2} .
$$

In what follows, we estimate $\left\|U_{l}^{s} f\right\|_{2}$ for each $s$. Set

$$
m_{j}^{l, s}(\xi)=\widehat{K_{j, s}}(\xi) \phi\left(2^{j-l} \xi\right)=m_{j, s}(\xi) \phi\left(2^{j-l} \xi\right) .
$$

And let $\bar{T}_{j}^{l, s}$ be the operator defined by

$$
\widehat{\bar{T}_{j}^{l, s} f}(\xi)=m_{j}^{l, s}\left(2^{-j} \xi\right) \hat{f}(\xi)
$$


By (ii) and (iii) of Lemma 3, we may assume, without loss of generality, that the support $Q_{s}$ of $b_{s}$ are uniformly small such that $\left|Q_{s}\right|<e^{\frac{q}{1-q}}$. Thus

$$
\left|m_{j, s}(\xi)\right|=\left|\widehat{K_{j, s}}(\xi)\right| \leq C\left|2^{j} \xi\right|^{\frac{1}{2 \log \left|Q_{s}\right|}} .
$$

By a straightforward computation, we get

$$
\left|\nabla m_{j, s}(\xi)\right|=\left|\nabla \widehat{K_{j, s}}(\xi)\right| \leq C 2^{j}
$$

So

$$
\left|m_{j}^{l, s}\left(2^{-j} \xi\right)\right|=\left|m_{j, s}\left(2^{-j} \xi\right) \phi\left(2^{-l} \xi\right)\right| \leq C 2^{\frac{l}{2 \log \left|Q_{s}\right|}}
$$

and

$$
\left|\nabla m_{j}^{l, s}\left(2^{-j} \xi\right)\right|=\left|\nabla\left(m_{j, s}\left(2^{-j} \xi\right) \phi\left(2^{-l} \xi\right)\right)\right| \leq C .
$$

By Lemma 2 again, there exists some constant $0<\theta<1$ such that

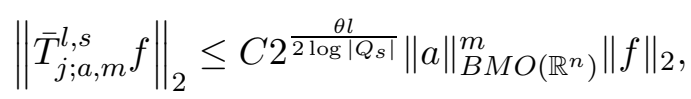

which by dilation-invariance implies

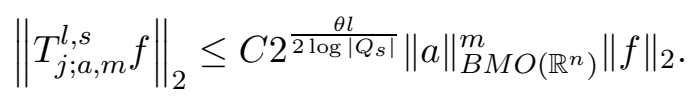

From this and Lemma 1(a), we obtain

$$
\begin{gathered}
\left\|\left(\sum_{j \in \mathbb{Z}}\left|\left(T_{j}^{l, s} S_{l-j}\right)_{a, m} f\right|^{2}\right)^{\frac{1}{2}}\right\|_{2}^{2} \\
\leq C \sum_{i=0}^{m}\left\|\left(\sum_{j \in \mathbb{Z}}\left|T_{j ; a, i}^{l, s}\left(S_{l-j ; a, m-i} f\right)\right|^{2}\right)^{\frac{1}{2}}\right\|_{2}^{2} \\
\leq C \sum_{i=0}^{m}\|a\|_{B M O\left(\mathbb{R}^{n}\right)^{2 i}}\left\|^{\frac{\theta l}{\log \mid Q s} \mid} \sum_{j}\right\| S_{l-j ; a, m-i} f \|_{2}^{2} \\
\leq C 2^{\frac{\theta l}{\log \left|Q_{s}\right|}\|a\|_{B M O\left(\mathbb{R}^{n}\right)}^{2 m}\|f\|_{2}^{2} .}
\end{gathered}
$$

Thus

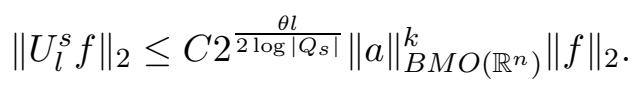


This shows that

$$
\begin{aligned}
\sum_{l>0}\left\|U_{l} f\right\|_{2} & \leq \sum_{s}\left|C_{s}\right| \sum_{l>0}\left\|U_{l}^{s} f\right\|_{2} \\
& \leq C \sum_{s}\left|C_{s}\right| \sum_{l>0} 2^{\frac{\theta l}{2 \log \left|Q_{s}\right|}\|a\|_{B M O\left(\mathbb{R}^{n}\right)}^{k}\|f\|_{2}} \\
& \leq C \sum_{s}\left|C_{s}\right|\left(\log \frac{1}{\left|Q_{s}\right|}\right)\|a\|_{B M O\left(\mathbb{R}^{n}\right)}^{k}\|f\|_{2} .
\end{aligned}
$$

Therefore, it follows from (2.6) and (2.10) that

$$
\left\|T_{a, k} f\right\|_{2} \leq \sum_{l \leq 0}\left\|U_{l} f\right\|_{2}+\sum_{l>0}\left\|U_{l} f\right\|_{2} \leq C\|a\|_{B M O\left(\mathbb{R}^{n}\right)}^{k}\|f\|_{2} .
$$

This completes the proof of Theorem 1.

\section{§3. Proof of Theorem 3}

The proof of Theorem 3 is based on the following two lemmas.

Lemma 4. Let $m$ be a positive number, $1<p<\infty$. If $a \in B L O\left(\mathbb{R}^{n}\right)$ and $a(x)$ is a subharmonic function, then the operator $M_{a, m}$ defined by

$$
M_{a, m} f(x)=\sup _{r>0} \frac{1}{r^{n}} \int_{|x-y| \leq r}|a(x)-a(y)|^{m}|f(y)| d y
$$

satisfies

$$
\left\|M_{a, m} f\right\|_{p} \leq C\|a\|_{B L O\left(\mathbb{R}^{n}\right)}^{m}\|f\|_{p}
$$

Note that for any cube $Q,|Q|^{-1} \int_{Q}\left|a(x)-a_{Q}\right|^{m} d x \leq\|a\|_{B L O\left(\mathbb{R}^{n}\right)}^{m}$. Since $a$ is a subharmonic function, this lemma follows from the same argument as in the proof of Theorems 2.3 and 2.4 in [8]. We omit the details.

Lemma 5. Let $\Omega_{0}$ be homogeneous of degree zero on $\mathbb{R}^{n}, 1<p<\infty$, a and $h$ be as in Theorem 2 . If $\Omega_{0} \in L^{\lambda}\left(S^{n-1}\right)$, for $\lambda>1$, then the operator

$$
M_{a, \tilde{m}}^{\Omega_{0}} f(x)=\sup _{r>0} \frac{1}{r^{n}} \int_{|x-y| \leq r}|a(x)-a(y)|^{\widetilde{m}}\left|h(|x-y|) \Omega_{0}(x-y) f(y)\right| d y
$$

satisfies

$$
\left\|M_{a, \widetilde{m}}^{\Omega_{0}} f\right\|_{p} \leq C\|a\|_{B L O\left(\mathbb{R}^{n}\right)}^{\widetilde{m}}\left\|\Omega_{0}\right\|_{L^{\lambda}\left(S^{n-1}\right)}\|f\|_{p}
$$

for all integer $\widetilde{m} \geq 0$. Here $C$ is independent of $\lambda$. 
Proof. For $\widetilde{m}=0$, Lemma 5 was proved by Calderón and Zygmund [5]. Next, we consider the case, $\widetilde{m}>0$. For any $\lambda>1$, write $\lambda^{\prime}=\frac{\lambda}{\lambda-1}$. Then by a double application of Hölder's inequality, we have

$$
\begin{aligned}
\left\|M_{a, \tilde{m}}^{\Omega_{0}} f\right\|_{p}^{p} & \leq\|h\|_{\infty}^{p} \int_{\mathbb{R}^{n}}\left(M_{a, \lambda^{\prime} \tilde{m}} f(x)\right)^{\frac{p}{\lambda^{\prime}}}\left(M_{\Omega_{0}^{\lambda}} f(x)\right)^{\frac{p}{\lambda}} d x \\
& \leq C\left\|M_{a, \lambda^{\prime} \tilde{m}} f\right\|_{p}^{\frac{p}{\lambda^{\prime}}}\left\|M_{\Omega_{0}^{\lambda}} f\right\|_{p}^{\frac{p}{\lambda}}
\end{aligned}
$$

where

$$
M_{\Omega_{0}^{\lambda}} f(x)=\sup _{r>0} \frac{1}{r^{n}} \int_{|x-y| \leq r}\left|\Omega_{0}^{\lambda}(x-y) f(y)\right| d y .
$$

It follows from Lemma 4 that

$$
\left\|M_{a, \lambda^{\prime} \widetilde{m}} f\right\|_{p^{\frac{p}{\lambda^{\prime}}}} \leq C\|a\|_{B L O\left(\mathbb{R}^{n}\right)}^{\widetilde{m} p}\|f\|_{p^{\frac{p}{\lambda^{\prime}}}}
$$

By the method of rotation of Calderón-Zygmund [5], it yields that

$$
\left\|M_{\Omega_{0}^{\lambda}} f\right\|_{p}^{\frac{p}{\lambda}} \leq C\left\|\Omega_{0}\right\|_{L^{\lambda}\left(S^{n-1}\right)}^{p}\|f\|_{p}^{\frac{p}{\lambda}} .
$$

Combining these estimates above, we complete the proof Lemma 5.

Proof of Theorem 3. By Definitions 1 and 2, we write $\Omega\left(y^{\prime}\right)=$ $\sum_{s} C_{s} b_{s}\left(y^{\prime}\right)$, where each $b_{s}$ is a $q$-block supported in $Q_{s}$. Thus

$$
\begin{aligned}
& M_{a, m}^{\Omega} f(x) \\
& \quad \leq \sum_{s}\left|C_{s}\right| \sup _{r>0} \int_{|x-y| \leq r}|a(x)-a(y)|^{m}\left|h(|x-y|) b_{s}(x-y) f(y)\right| d y \\
& \quad:=\sum_{s}\left|C_{s}\right| M_{a, m}^{b_{s}} f(x) .
\end{aligned}
$$

Consequently,

$$
\left\|M_{a, m}^{\Omega} f\right\|_{p} \leq \sum_{s}\left|C_{s}\right|\left\|M_{a, m}^{b_{s}} f\right\|_{p} .
$$

We now estimate $\left\|M_{a, m}^{b_{s}} f\right\|_{p}$ for each $b_{s}$. It follows from Lemma 5 that for any $\lambda>1$,

$$
\left\|M_{a, m}^{b_{s}} f\right\|_{p} \leq C\left\|b_{s}\right\|_{L^{\lambda}\left(S^{n-1}\right)}\|a\|_{B L O\left(\mathbb{R}^{n}\right)}^{m}\|f\|_{p} .
$$

Notice that $\operatorname{supp}\left(b_{s}\right) \subseteq Q_{s}$ and $\left\|b_{s}\right\|_{L^{q}\left(S^{n-1}\right)} \leq\left|Q_{s}\right|^{\frac{1}{q}-1}$. If $\left|Q_{s}\right| \geq e^{\frac{q}{1-q}}$, we let $\lambda=q$, to get

$$
\begin{aligned}
\left\|M_{a, m}^{b_{s}} f\right\|_{p} & \leq C\left\|b_{s}\right\|_{L^{q}\left(S^{n-1}\right)}\|a\|_{B L O\left(\mathbb{R}^{n}\right)}^{m}\|f\|_{p} \\
& \leq C\left|Q_{s}\right|^{\frac{1}{q}-1}\|a\|_{B L O\left(\mathbb{R}^{n}\right)}^{m}\|f\|_{p} \leq C\|a\|_{B L O\left(\mathbb{R}^{n}\right)}^{m}\|f\|_{p} .
\end{aligned}
$$


If $\left|Q_{s}\right|<e^{\frac{q}{1-q}}$, let $\lambda=\log \left|Q_{s}\right| /\left(1+\log \left|Q_{s}\right|\right)$, so that $1<\lambda<q$ and $\lambda^{\prime}=-\log \left|Q_{s}\right|$. By Hölder's inequality, we have

$$
\begin{aligned}
\left\|M_{a, m}^{b_{s}} f\right\|_{p} & \leq C\left\|b_{s}\right\|_{L^{q}\left(S^{n-1}\right)}\left|Q_{s}\right|^{\frac{1}{\lambda}-\frac{1}{q}}\|a\|_{B L O\left(\mathbb{R}^{n}\right)}^{m}\|f\|_{p} \\
& \leq C\left|Q_{s}\right|^{-\frac{1}{\lambda^{\prime}}}\|a\|_{B L O\left(\mathbb{R}^{n}\right)}^{m}\|f\|_{p} \leq C\|a\|_{B L O\left(\mathbb{R}^{n}\right)}^{m}\|f\|_{p} .
\end{aligned}
$$

So, we obtain

$$
\left\|M_{a, m}^{\Omega} f\right\|_{p} \leq C \sum_{s}\left|C_{s}\right|\|a\|_{B L O\left(\mathbb{R}^{n}\right)}^{m}\|f\|_{p} \leq C\|a\|_{B L O\left(\mathbb{R}^{n}\right)}^{m}\|f\|_{p}
$$

and complete the proof of Theorem 3.

\section{§4. Proof of Theorem 2}

To prove Theorem 2, we still need the following auxiliary result.

Let $h, a, k$ and $\Omega\left(y^{\prime}\right)=\sum_{s} C_{s} b_{s}\left(y^{\prime}\right)$ be as in Theorem $2, j \in \mathbb{Z}$. Define the following operators:

$$
\begin{aligned}
\sigma_{j ; a, k} f(x) & =\int_{2^{j}<|x-y| \leq 2^{j+1}}[a(x)-a(y)]^{k} \frac{\Omega(x-y)}{|x-y|^{n}} h(|x-y|) f(y) d y, \\
\sigma_{j ; a, k}^{s} f(x) & =\int_{2^{j}<|x-y| \leq 2^{j+1}}[a(x)-a(y)]^{k} \frac{b_{s}(x-y)}{|x-y|^{n}} h(|x-y|) f(y) d y, \\
\mu_{j ; a, k} f(x) & =\int_{2^{j}<|x-y| \leq 2^{j+1}}|a(x)-a(y)|^{k} \frac{|\Omega(x-y)|}{|x-y|^{n}}|h(|x-y|)| f(y) d y, \\
\mu_{j ; a, k}^{s} f(x) & =\int_{2^{j}<|x-y| \leq 2^{j+1}}|a(x)-a(y)|^{k} \frac{\left|b_{s}(x-y)\right|}{|x-y|^{n}}|h(|x-y|)| f(y) d y, \\
\mu_{a, k}^{*} f(x) & =\sup _{j \in \mathbb{Z}}\left|\mu_{j ; a, k} f(x)\right| \text { and } \mu_{a, k}^{s *} f(x)=\sup _{j \in \mathbb{Z}}\left|\mu_{j ; a, k}^{s} f(x)\right| .
\end{aligned}
$$

Clearly, we have

$$
\mu_{a, k}^{*} f(x) \leq C M_{a, k}^{\Omega} f(x) \text { and } \mu_{a, k}^{s *} f(x) \leq C M_{a, k}^{b_{s}} f(x) .
$$

By Lemma 5 and Theorem 3, it is easy to see that for all $1<p<\infty$,

$$
\left\|\mu_{a, k}^{*} f\right\|_{p} \leq C\|a\|_{B L O\left(\mathbb{R}^{n}\right)}^{k}\|f\|_{p}
$$

and

$$
\left\|\mu_{a, k}^{s *} f\right\|_{p} \leq C\left\|b_{s}\right\|_{L^{\lambda}\left(S^{n-1}\right)}\|a\|_{B L O\left(\mathbb{R}^{n}\right)}^{k}\|f\|_{p},
$$

and the bounds are independent of $b_{s}$.

By applying (4.1) and (4.2), we can obtain the following lemma. 
LEMMA 6. Under the same assumptions as in Theorem 2, for arbitrary functions $f_{j}$,

$$
\left\|\left(\sum_{j \in \mathbb{Z}}\left|\sigma_{j ; a, k} f_{j}\right|^{2}\right)^{\frac{1}{2}}\right\|_{p} \leq C\|a\|_{B L O\left(\mathbb{R}^{n}\right)}^{k}\left\|\left(\sum_{j \in \mathbb{Z}}\left|f_{j}\right|^{2}\right)^{\frac{1}{2}}\right\|_{p}
$$

and

$$
\begin{gathered}
\left\|\left(\sum_{j \in \mathbb{Z}}\left|\sigma_{j ; a, k}^{s} f_{j}\right|^{2}\right)^{\frac{1}{2}}\right\|_{p} \\
\leq C\left\|b_{s}\right\|_{L^{\lambda}\left(S^{n-1}\right)}\|a\|_{B L O\left(\mathbb{R}^{n}\right)}^{k}\left\|\left(\sum_{j \in \mathbb{Z}}\left|f_{j}\right|^{2}\right)^{\frac{1}{2}}\right\|_{p}
\end{gathered}
$$

for all $1<p<\infty$ and for any $\lambda>1$.

Proof. We prove only (4.3) because the other is essentially similar. The ideas in our proof are taken from those in Lemma of [7] and Lemma 2 of [11]. In fact, it suffices to consider the case $p>2$ so that $q=\left(\frac{p}{2}\right)^{\prime}$, and there exists $g \in L_{+}^{q}$ of unit norm such that

$$
\left\|\left(\sum_{j \in \mathbb{Z}}\left|\sigma_{j ; a, k} f_{j}\right|^{2}\right)^{\frac{1}{2}}\right\|_{p}^{2}=\int_{\mathbb{R}^{n}} \sum_{j \in \mathbb{Z}}\left|\sigma_{j ; a, k} f_{j}(x)\right|^{2} g(x) d x .
$$

Also, by Hölder's inequality and a simple computation, we have

$$
\left|\sigma_{j ; a, k} f(x)\right|^{2} \leq C \mu_{j ; a, 2 k}\left(|f|^{2}\right)(x)
$$

and

$$
\int_{\mathbb{R}^{n}} \mu_{j ; a, k}\left(|f|^{2}\right)(x) g(x) d x=\int_{\mathbb{R}^{n}} f^{2}(x) \mu_{j ; \tilde{a}, 2 k} \widetilde{g}(-x) d x
$$


where $\widetilde{a}(x)=a(-x)$ and $\widetilde{g}(x)=g(-x)$. Therefore

$$
\begin{aligned}
\left\|\left(\sum_{j \in \mathbb{Z}}\left|\sigma_{j ; a, k} f_{j}\right|^{2}\right)^{\frac{1}{2}}\right\|_{p}^{2} & \leq C \int_{\mathbb{R}^{n}} \sum_{j \in \mathbb{Z}} \mu_{j ; a, 2 k}\left(\left|f_{j}\right|^{2}\right)(x) g(x) d x \\
& =C \int_{\mathbb{R}^{n}} \sum_{j \in \mathbb{Z}} f_{j}^{2}(x) \mu_{j ; \widetilde{a}, 2 k} \widetilde{g}(-x) d x \\
& \leq C \int_{\mathbb{R}^{n}} \sup _{j \in \mathbb{Z}}\left|\mu_{j ; \widetilde{a}, 2 k} \widetilde{g}(-x)\right| \sum_{j \in \mathbb{Z}} f_{j}^{2}(x) d x \\
& \leq C\left\|\mu_{\widetilde{a}, 2 k}^{*} \widetilde{g}\right\|_{q}\left\|\sum_{j \in \mathbb{Z}}\left|f_{j}\right|^{2}\right\|_{\frac{p}{2}} .
\end{aligned}
$$

By (4.1), we obtain

$$
\begin{gathered}
\left\|\left(\sum_{j \in \mathbb{Z}}\left|\sigma_{j ; a, k} f_{j}\right|^{2}\right)^{\frac{1}{2}}\right\|_{p}^{2} \leq C\|a\|_{B L O\left(\mathbb{R}^{n}\right)}^{2 k}\|g\|_{q}\left\|\left(\sum_{j \in \mathbb{Z}}\left|f_{j}\right|^{2}\right)^{\frac{1}{2}}\right\|_{p}^{2} \\
=C\|a\|_{B L O\left(\mathbb{R}^{n}\right)}^{2 k}\left\|\left(\sum_{j \in \mathbb{Z}}\left|f_{j}\right|^{2}\right)^{\frac{1}{2}}\right\|_{p}^{2},
\end{gathered}
$$

which proves Lemma 6.

Proof of Theorem 2. Let $U_{l}, T_{j}^{l}, S_{l-j}$ be the same as that in the proof of Theorem 1. Then for $1<p<\infty$, similarly to (2.1) and (2.2), we have

$$
\left\|T_{a, k} f\right\|_{p} \leq \sum_{l \in \mathbb{Z}}\left\|U_{l} f\right\|_{p}
$$

and

$$
\left\|U_{l} f\right\|_{p} \leq C \sum_{m=0}^{k}\|a\|_{B L O\left(\mathbb{R}^{n}\right)}^{k-m}\left\|\left(\sum_{j \in \mathbb{Z}}\left|\left(T_{j}^{l} S_{l-j}\right)_{a, m} f\right|^{2}\right)^{\frac{1}{2}}\right\|_{p}
$$

Now we estimate $\left\|U_{l} f\right\|_{p}$ in two cases as follows: 
Case 1. First we show the $L^{p}$-boundedness of $U_{l}$ for $l \leq 0$.

For $p=2$, by the same arguments as to (2.6), we obtain

$$
\left\|U_{l} f\right\|_{2} \leq C 2^{v l}\|a\|_{B L O\left(\mathbb{R}^{n}\right)}^{k}\|f\|_{2} .
$$

Next we turn to estimate $L^{p}$-boundedness of $U_{l} f$. Write

$$
\left(T_{j}^{l} S_{l-j}\right)_{a, m} f(x)=\sum_{i=0}^{m} C_{m}^{i} \sigma_{j ; a, i}\left(S_{l-j ; a, m-i}^{2} f\right)(x) .
$$

We know from Lemma 6 and Lemma 1(b) that for all $1<p<\infty$,

$$
\begin{aligned}
\left\|U_{l} f\right\|_{p} & \leq C \sum_{m=0}^{k}\|a\|_{B L O\left(\mathbb{R}^{n}\right)}^{k-m} \sum_{i=0}^{m}\left\|\left(\sum_{j \in \mathbb{Z}}\left|\sigma_{j ; a, i}\left(S_{l-j ; a, m-i}^{2} f\right)\right|^{2}\right)^{\frac{1}{2}}\right\|_{p} \\
& \leq C \sum_{m=0}^{k} \sum_{i=0}^{m} C_{i}^{m}\|a\|_{B L O\left(\mathbb{R}^{n}\right)}^{k-m+i}\left\|\left(\sum_{j \in \mathbb{Z}}\left|S_{l-j ; a, m-i}^{2} f\right|^{2}\right)^{\frac{1}{2}}\right\|_{p} \\
& \leq C\|a\|_{B L O\left(\mathbb{R}^{n}\right)}^{k}\|f\|_{p} .
\end{aligned}
$$

Using interpolation between (4.7) and (4.8), we obtain

$$
\sum_{l \leq 0}\left\|U_{l} f\right\|_{p} \leq C\|a\|_{B L O\left(\mathbb{R}^{n}\right)}^{k}\|f\|_{p}
$$

Case 2. We next consider the $L^{p}$-estimate of $U_{l}$ for $l>0$.

Let $T_{j, s}^{l}, S_{l-j}, U_{l}^{s}$ be as that in the proof of Theorem 1. Similarly to (2.7) and (2.8), we have for $1<p<\infty$,

$$
\left\|U_{l} f\right\|_{p} \leq \sum_{s}\left|C_{s}\right|\left\|U_{l}^{s} f\right\|_{p}
$$

and

$$
\left\|U_{l}^{s} f\right\|_{p} \leq C \sum_{m=0}^{k}\|a\|_{B L O\left(\mathbb{R}^{n}\right)}^{k-m}\left\|\left(\sum_{j \in \mathbb{Z}}\left|\left(T_{j}^{l, s} S_{l-j}\right)_{a, m} f\right|^{2}\right)^{\frac{1}{2}}\right\|_{p}
$$


For each $b_{s}$, without loss of generality, we may assume that the support $Q_{s}$ of $b_{s}$ are uniformly small such that $\left|Q_{s}\right|<e^{\frac{q}{1-q}}$. Similarly to (2.9), we can get that for some $0<\theta<1$,

$$
\left\|U_{l}^{s} f\right\|_{2} \leq C 2^{\frac{\theta l}{2 \log \left|Q_{s}\right|}}\|a\|_{B L O\left(\mathbb{R}^{n}\right)}^{k}\|f\|_{2} .
$$

For $1<p<\infty$, noting that

$$
\left(T_{j}^{l, s} S_{l-j}\right)_{a, m} f(x)=\sum_{i=0}^{m} C_{m}^{i} \sigma_{j ; a, i}^{s}\left(S_{l-j ; a, m-i}^{2} f\right)(x)
$$

and invoking (4.4) and Lemma 1(b) with $\lambda=\frac{\log \left|Q_{s}\right|}{1+\log \left|Q_{s}\right|}$, we have

$$
\begin{aligned}
\left\|U_{l}^{s} f\right\|_{p} & \leq C \sum_{m=0}^{k}\|a\|_{B L O\left(\mathbb{R}^{n}\right)}^{k-m} \sum_{i=0}^{m}\left\|\left(\sum_{j \in \mathbb{Z}}\left|\sigma_{j ; a, i}^{s}\left(S_{l-j ; a, m-i}^{2} f\right)\right|^{2}\right)^{\frac{1}{2}}\right\|_{p} \\
& \leq C\left\|b_{s}\right\|_{L^{\lambda}\left(S^{n-1}\right)} \sum_{m=0}^{k} \sum_{i=0}^{m}\|a\|_{B L O\left(\mathbb{R}^{n}\right)}^{k-m+i}\left\|\left(\sum_{j \in \mathbb{Z}}\left|S_{l-j ; a, m-i}^{2} f\right|^{2}\right)^{\frac{1}{2}}\right\|_{p} \\
& \leq C\|a\|_{B L O\left(\mathbb{R}^{n}\right)}^{k}\|f\|_{p} .
\end{aligned}
$$

Using interpolation between (4.12) and (4.13) again, we obtain

$$
\left\|U_{l}^{s} f\right\|_{p} \leq C 2^{\frac{\theta_{1} \theta l}{\log \left|Q_{s}\right|}}\|a\|_{B L O\left(\mathbb{R}^{n}\right)}^{k}\|f\|_{p},
$$

for some $0<\theta_{1} \leq 1$. This shows that

$$
\begin{aligned}
\sum_{l>0}\left\|U_{l} f\right\|_{p} & \leq \sum_{s}\left|C_{s}\right| \sum_{l>0}\left\|U_{l}^{s} f\right\|_{p} \\
& \leq C \sum_{s}\left|C_{s}\right| \sum_{l>0} 2^{\frac{\theta_{1} \theta l}{2 \log \left|Q_{s}\right|}}\|a\|_{B L O\left(\mathbb{R}^{n}\right)}^{k}\|f\|_{p} \\
& \leq C \sum_{s}\left|C_{s}\right|\left(\log \frac{1}{\left|Q_{s}\right|}\right)\|a\|_{B L O\left(\mathbb{R}^{n}\right)}^{k}\|f\|_{p} .
\end{aligned}
$$

Therefore, (4.9) and (4.15) now imply

$$
\left\|T_{a, k} f\right\|_{p} \leq \sum_{l \leq 0}\left\|U_{l} f\right\|_{p}+\sum_{l>0}\left\|U_{l} f\right\|_{p} \leq C\|a\|_{B L O\left(\mathbb{R}^{n}\right)}^{k}\|f\|_{p},
$$

which completes the proof of Theorem 2 . 
Acknowledgements. The authors would like to express their gratitude to the referee for his very valuable comments.

\section{REFERENCES}

[1] A.J. AL-Hasan and D.S. Fan, A singular integral operator related block spaces, Hokkaido Math. J., 28 (1999), 285-299.

[2] J. Alvarez, R. Bagby, D. Kurtz and C. Pérez, Weighted estimates for commutators of linear operators, Studia Math., 104 (1993), 195-209.

[3] R. Coifman and R. Rochberg, Another characterization of BMO, Proc. Amer. Math. Soc., 79(2) (1980), 249-254.

[4] R. Coifman, R. Rochberg and G. Weiss, Factorization theorems for Hardy spaces in several variables, Ann. of Math., 103 (1976), 611-636.

[5] A.P. Calderon and A. Zygmund, On singular integrals, Amer. J. Math., 78 (1956), 289-309.

[6] J. Duoandikoetxea, Weighted norm inequalities for homogeneous singular integrals, Trans. Amer. Math. Soc., 336 (1993), 869-880.

[7] J. Duoandikoetxea and J.L. Rubio de Francia, Maximal and singular integral operators via Fourier transform estimates, Invent. Math., 84 (1996), 541-561.

[8] J. Garcia-Cuerva, E. Harboure, C. Segovia and J.L. Torre, Weighted norm inequalities for commutators of strongly singular integrals, Indiana Univ. Math. J., 40 (1991), $1397-1420$.

[9] G.E. Hu, $L^{2}$-boundedness for the commutators with convolution operators, Nagoya Math.J., 163 (2001), 55-70.

[10] Weighted norm inequalities for commutators of homogeneous singular integral operators, Acta Math. Sinica, New series, 11 (1995), Special Issue: 77-88.

[11] G.E. Hu, S.Z. Lu and B.L. Ma, The commutators of convolution operators (in Chinese), Acta Math. Sinica, 42 (1999), 359-368.

[12] G.E. Hu and B.L. Ma, $L^{2}\left(\mathbb{R}^{n}\right)$ boundedness for the commutators of homogeneous singular integral operators, Acta Math. Sinica (to appear).

[13] G.E. Hu and Q.Y. Sun, $L^{p}$ boundedness for the commutators of convolution operators, Chinese Ann. of Math. (Ser. A), 22(4) (2001), 509-516.

[14] M. Keitoku and S. Sato, Block spaces on the unit sphere in $\mathbb{R}^{n}$, Proc. Amer. Math. Soc., 119 (1993), 453-455.

[15] S.Z. Lu, M. Taibleson and G. Weiss, Spaces Generated by Blocks, Beijing Normal University Press, Beijing, 1989.

[16] S.Z. Lu and H.X. Wu, Oscillatory singular integrals and commutators with rough kernels, Ann. Sci. Math. Québec, 27(1) (2003), 47-66.

[17] M.H. Taibleson and G. Weiss, Certain function spaces associated with a.e. convergence of Fourier series, Univ. of Chicago Conf. in honor of Zygmund, Vol.I, Woodsworth, 1983, 95-113. 
Shanzhen Lu

Department of Mathematics

Beijing Normal University

Beijing, 100875

P. R. China

lusz@bnu.edu.cn

Huoxiong $\mathrm{Wu}$

School of Mathematical Sciences

Xiamen University

Xiamen, Fujian 361005

China

huoxwu@xmu.edu.cn 